\title{
Repair Using Conventional Implant for Ruptured Annulus Fibrosus after Lumbar Discectomy: Surgical Technique and Case Series
}

\author{
Bo-Gun Suh ${ }^{1}$, Jae-Hyung Uh ${ }^{1}$, Sang-Hyuk Park ${ }^{2}$, Gun Woo Lee ${ }^{3}$ \\ ${ }^{1}$ Spine Center and Department of Orthopaedic Surgery, Pohang Semyeng Christianty Hospital, Pohang, Korea \\ ${ }^{2}$ Spine Center and Department of Neurosurgery, Pohang Semyeng Christianty Hospital, Pohang, Korea \\ ${ }^{3}$ Department of Orthopaedic Surgery, Armed Forces Yangju Hospital, Yangju, Korea
}

Study Design: A retrospective review of annulus fibrosus repair (AR) using a novel technique with a conventional implant.

Purpose: The purpose of this study was to present the feasibility and clinico-radiological outcomes of a novel AR technique using a conventional implant to minimize recurrence following a lumbar discectomy (LD).

Overview of Literature: Conventional repair techniques to prevent recurrence following LD have several drawbacks. The AR surgical technique has received little attention as an adjunct to LD.

Methods: A total of 19 patients who underwent novel AR following LD, and who were available for follow-up for at least three years, were enrolled in this study. Several variables, including the type and size of disc herniation, and the degree of disc degeneration, were evaluated preoperatively. Postoperatively, the presence of clinical and radiological recurrence of disc herniation was evaluated from pain intensity and functional statuses, as well as an enhanced L-spine magnetic resonance imaging at the final follow-up. The presence of a peripheral hollow rim and inserted anchor mobilization were also evaluated during the follow-up.

Results: During follow-ups, there were no recurrences of disc herniation or complications, including neurovascular complications. Pain and functional disability improved significantly after surgery, and the improvement was maintained throughout the three-year follow-up period. No mobilization or implant peripheral hollow rim was observed during the follow-up.

Conclusions: This study examined the feasibility of a novel and easily available annulus implant technique following LD. These results suggest performing AR with this technique may be a valuable alternative for optimizing outcomes, if the procedure is performed in proper candidates.

Keywords: Annulus fibrosus; Repair; Intervertebral disc displacement; Lumbar vertebrae

\section{Introduction}

Although lumbar discectomy (LD) yields improvements in pain and physical function as well as a decrease in disability for the majority of patients with lumbar disc herniation (LDH), same-level recurrent lumbar disc herniation $(\mathrm{rLDH})$, which is reported to have an incidence of approximately $3 \%-23 \%$, complicates favorable outcomes [1-7]. Consequently, determining the causative factors of rLDH after LD and finding ways to overcome this prob-

Received Feb 25, 2014; Revised Sep 7, 2014; Accepted Sep 9, 2014

Corresponding author: Gun Woo Lee

Department of Orthopaedic Surgery, Armed Forces Yangju Hospital,

461 Yongam-ri, Eunhyeon-myeon, Yangju, Korea

Tel: +82-31-787-7195, Fax: +82-2-787-4056, E-mail: gwlee1871@gmail.com 
lem are necessary because patients with rLDH may eventually become symptomatic and require further operative intervention.

The literature on techniques to prevent rLDH rarely includes modifications of the LD technique $[1,4,5]$, although the amount of discectomy could be a critical factor $[1,2,4,7]$. Sub-total discectomy, a highly aggressive removal procedure, has been criticized for causing nucleus pulposus and endplate injury, resulting in accelerated degenerative disc change and a loss of disc height. This is potentially associated with an increase in the incidence of chronic back pain. However, several reports have also concluded this aggressive technique is associated with a lower rLDH incidence at the affected level [2-6,8]. Alternately, limited discectomy removes only the sequestrated disc, potentially leaving part of the disc to compress the dura and nerve root. This has also been criticized due to its association with a higher rLDH incidence, although this limited surgery may reduce degenerative disc changes and decrease persistent back pain [2-6,8]. That is, performing a minimal removal of just the herniated disc irritating the nerve, along with certain modifications to the surgical technique to avoid $\mathrm{rLDH}$, can be achieved and produce an ideal outcome.

Annulus fibrosus repair (AR) has been sparsely reported as an adjunct modification to LD for minimizing recurrence or disastrous complication $[9,10]$, and seemed to provide a relatively simple approach for spine surgeons. A few studies of AR have shown that it can be an effective way to lower the recurrence rate and improve annulus healing [7,9-11]. However, several conventional techniques for AR have drawbacks: (1) it is technically demanding and difficult to perform the repair in a confined and deeply-seated surgical space; (2) there is potential for suture knots to protrude into the spinal canal and irritate the nerve along with the existing herniated disc, thereby creating persistent back pain and radiating leg pain; (3) these techniques are insufficient to provide mechanical strength against intradiscal pressure and annulus fibrosus (AF) tensile forces; and (4) although there are a few commercially available implants for AR, they are quite expensive and unavailable worldwide.

To our knowledge, AR to lower rLDH incidence after $\mathrm{LD}$, and a knotless suture technique utilizing an easily available commercial implant has not previously been reported. The purpose of this study was to determine the feasibility of applying a conventional, widely available implant during $\mathrm{LD}$, and to evaluate the potential benefits of this special technique in terms of surgical outcome and recurrence. It was postulated that repair of an annulotomy or spontaneously ruptured AF using this technique would stabilize disc material, thereby reducing the incidence of recurrent herniation as well as affected disc degeneration.

\section{Materials and Methods}

\section{Inclusion and exclusion criteria}

This study received the approval of the Institutional Review Board of Pohang Semyeng Christianty Hospital.

This study was designed as a retrospective review of medical records between January 2007 and January 2008. A total of 19 consecutive patients who underwent AR following LD, and who were able to be followed-up for at least three years were enrolled in this study. No. 2 fiberwire sutures and PushLock implants (Arthrex, Naples, FL, USA) were used in all cases. All operations were performed by a single surgeon (the corresponding author). Indications that a patient required $\mathrm{AR}$ after a discectomy were: (1) intractable pain that did not respond to conservative treatment over 12 weeks in young patients; (2) development of neurological deficits confirmed by magnetic resonance image (MRI) of the lumbar spine; and (3) no history of prior surgery at the same level of the lumbar spine. Exclusion criteria included: (1) severe degenerative change or tethered margin of injured AF in spite of young age, (2) herniated disc of a foraminal or extraforaminal location on MRI, and (3) any spinal disease other than disc herniation, such as spinal stenosis.

\section{Surgical technique and postoperative management}

All surgeries were performed with the patient in a prone position. A typical midline skin incision, approximately 3-4 cm long, was made over the affected level. A portion of the caudal part of the superior lamina was sometimes removed, but a medial facetectomy was performed rarely and only if the medial facet was clearly impinging on the nerve root after the discectomy. In cases of transligamentous extrusion (TLE) or sequestration, the most minimal herniated disc portion was removed after advertent preparation; this allowed for identification of the ruptured $\mathrm{AF}$ site. The ruptured AF site is almost always located on the 
posterolateral portion, which is near the lower endplate of the affected disc space (Fig. 1). In cases of subligamentous extrusion (SLE), annulotomy was performed as close as possible to the lower endplate after advertent preparation, and then the problematic disc was removed. The compressed nerve root was always examined along its course to the foramen, and, if necessary, a partial foraminotomy was also performed. Drainage was kept in all patients for one day.

AR procedures using the PushLock implant were relatively simple and easy. First, after completion of discectomy and decompression of the nerve root, the AF footprint center at the vertebral body corner was identified, and a hole at the center for inserting the implant was made. Second, the annulotomy edge or spontaneously ruptured AF site was sewn up with two fiber-wire sutures. Third, to improve ruptured AF healing, the AF footprint was carefully prepared and abraded near the corner of the vertebral body, not the endplate, by removing the covering soft tissue, and rasping with a curette until blood spots appeared. Finally, the AF edge was grasped by pulling in the direction of the bone bed, which positioned the

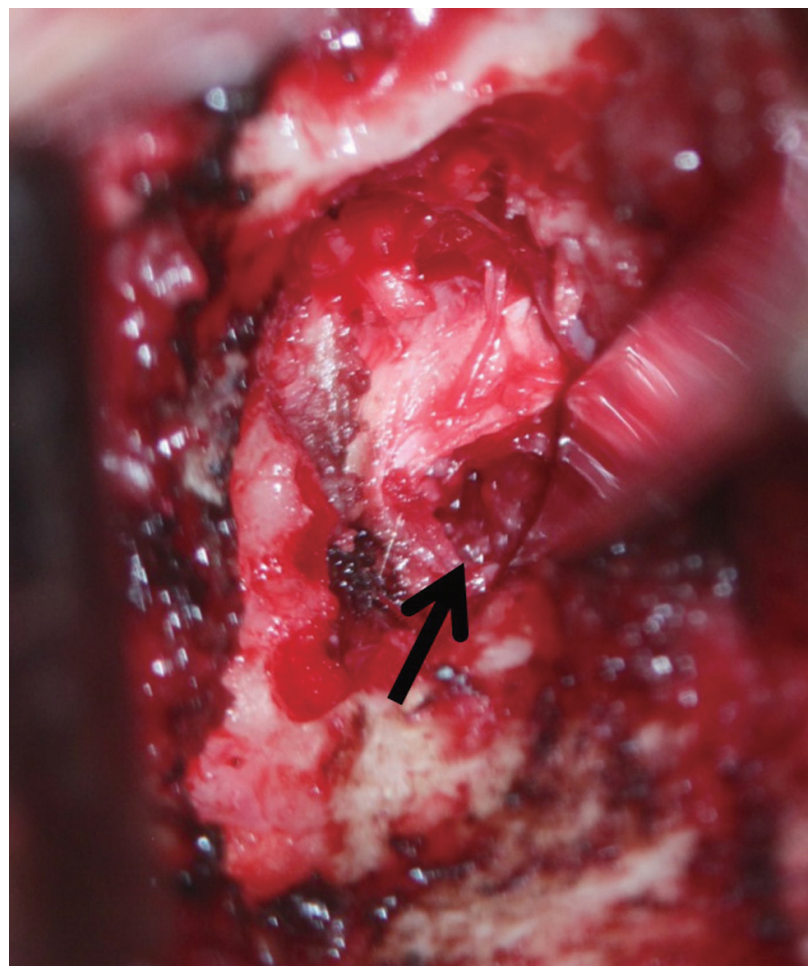

Fig. 1. Intraoperative photograph revealed that a spontaneous rupture of annulus fibrosis (black arrow) was detected at the posterolateral aspect near inferior endplate attaching the annulus fibrosus. edge at the footprint. Then, two sewn threads anchoring the implant were hammered into the cortical bone (Fig. 2).

Postoperative protocols were almost entirely the same as those generally performed after lumbar discectomy. All patients were allowed to ambulate the first postoperative day, and were discharged from the hospital on the second or third postoperative day. For one month following surgery, patients were not permitted to sit for long periods of time or lift heavy objects. Three months after surgery, patients were allowed to resume normal activities and exercise.

\section{Data collection and analysis}

Demographic data, clinical data, and radiological data were retrospectively collected from medical records. Following surgery, an assessment was performed at regular follow-up intervals immediately after surgery, at three and six months, one year, then annually thereafter for up to three years. For clinical data, the visual analog scale

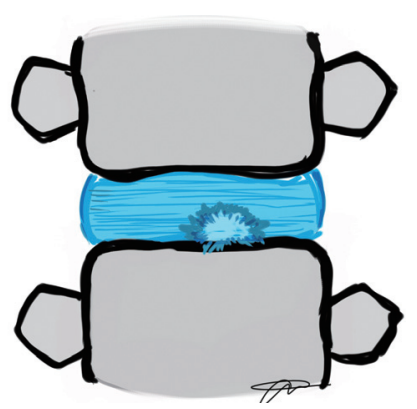

(A)
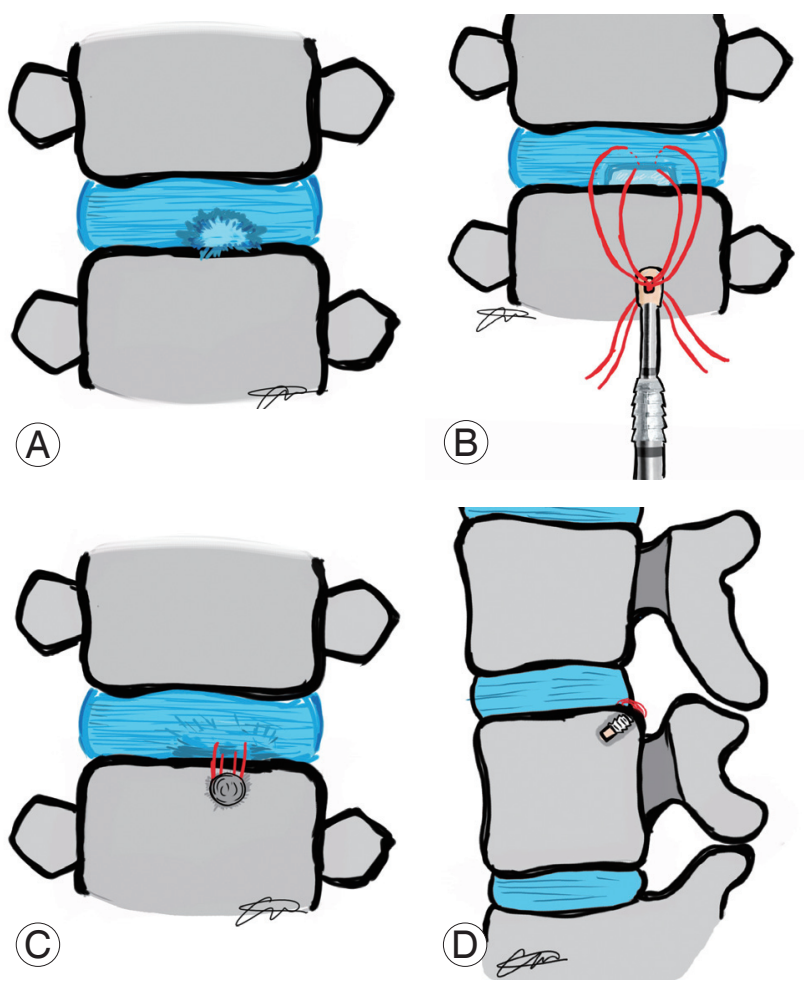

Fig. 2. Schematic of our surgical technique for repair of a ruptured annulus fibrosus. (A) Ruptured annulus fibrosus at the posterolateral aspect. (B) The edge of the annulotomy site or the spontaneously ruptured site was sewn with two fiber wire sutures. (C, D) The edge was grasped by pulling toward the bone bed and was positioned at the edge of the footprint. Then, the two sewn threads for anchoring the implant were hammered into the cortical bone. 
Table 1. The Pfirrmann magnetic resonance imaging classification of disc degeneration

\begin{tabular}{llcl} 
Grade & Signal intensity/structure of nucleus pulposus & $\begin{array}{c}\text { Distinction between } \\
\text { annulus and nucleus }\end{array}$ & Disc height \\
Grade I & Homogenous hyperintense (like CSF)/bright white & Clear & Normal \\
Grade II & In homogenous hyperintense/bright+horizontal band & Clear & Normal \\
Grade III & Intermediate/inhomogenous, gray & Unclear & Normal to moderately decreased \\
Grade IV & Intermediate-hypointense/inhomogenous, gray to black & Lost & Collapsed disc space \\
\hline Grade V & Hypointense/inhomogenous, black & Lost &
\end{tabular}

CSF, cerebrospinal fluid.

(VAS) and the Oswestry disability index (ODI) were used to assess patient pain and functional severity.

All preoperative MRIs were analyzed by one spine surgeon who evaluated the degree of disc degeneration at the affected lumbar segment using the PACS system (Infinitt, Bracknell, Berkshire, UK) by Pfirrmann et al. [12] (Table 1). At the final follow-up appointment, an enhanced L-spine MRI with gadolinium was performed on all enrolled patients to evaluate possible disc herniation recurrence.

$\mathrm{AP}$ and lateral radiographs were taken at each followup appointment. Special attention was paid to the presence of a peripheral hollow during follow-up, and the mobilization of the inserted anchor. Any changes in the PushLock implant were analyzed in a series of follow-up radiographs. The anterior and posterior heights of the affected disc were assessed before and at regular follow-up times after surgery.

\section{Statistical analysis}

Nonparametric statistical analysis was used due to the relatively small sample size. Statistical analyses were performed using the Mann-Whitney $U$ and Wilcoxon matched-pair signed-rank tests. SPSS ver. 19.0 (IBM Co., Armonk, NY, USA) was used to perform analyses. Statistical significance was defined as a two-tailed $p$-value of less than 0.05 .

\section{Results}

\section{Patient characteristics}

All patients were available for complete follow-up. There were 8 men and 11 women, and the mean age was 34.7 years (range, 26-47 years). All patients had a minimum follow-up duration of three years with a mean followup of 41.2 months (range, 37-46 months). Four patients experienced traumatic events. All patients underwent discectomy surgery targeting a single lumbar spine level. Disc herniation levels in this study were L3-L4 (2 cases), L4-L5 (11 cases), and L5-S1 (6 cases). The degree of disc degeneration described by Pfirrmann's classification is presented in Table 2.

\section{Surgical outcome and recurrence}

The mean procedure time was 0.8 hours (range, $0.6-1.0$ hours), and mean intraoperative blood loss was $50 \mathrm{~mL}$ (range, 30-60 mL). There were no recurrences of disc herniation or clinical complications, including neurovascular complications, during the follow-up period. Pain and functional disability improved significantly after surgery, and the improvement was maintained throughout the three-year follow-up. Clinical outcomes measured using the VAS and ODI are summarized in Table 3.

During regular follow-up after surgery, any symptoms or signs related to recurrent disc herniation were examined. In addition, at the three-year follow-up, the enhanced L-spine MRIs showed no re-herniated disc at the affected segments. No mobilization or implant peripheral hollow rim were observed during follow-up. Anterior and posterior disc heights at the last follow-up were also maintained, and were comparable to the heights measured before surgery. Radiologic outcomes, including disc heights, mobilization, and peripheral hollow rim, are summarized in Table 4.

\section{Discussion}

The incidence of rLDH following LD ranges from $3 \%$ to $23 \%[1,6,8]$. Recurrence can lead to physical deteriora- 
Table 2. Patients' characteristics

\begin{tabular}{|c|c|c|c|c|c|c|c|c|c|c|}
\hline \multirow[b]{2}{*}{ No } & \multirow[b]{2}{*}{ Sex } & \multirow{2}{*}{$\begin{array}{l}\text { Age } \\
\text { (yr) }\end{array}$} & \multirow[b]{2}{*}{ BMI } & \multirow[b]{2}{*}{ Comorbidity } & \multirow{2}{*}{$\begin{array}{l}\text { LDH } \\
\text { level }\end{array}$} & \multirow[b]{2}{*}{ LDH type } & \multicolumn{2}{|c|}{ AF tear } & \multirow{2}{*}{$\begin{array}{l}\text { Degree of } \\
\text { disc } \\
\text { degeneration }\end{array}$} & \multirow[b]{2}{*}{ Complication } \\
\hline & & & & & & & $\begin{array}{l}\text { Size } \\
(\mathrm{mm})\end{array}$ & Type & & \\
\hline 1 & Male & 37 & 27.8 & - & L4-5 & Extrusion & 5 & Annulotomy & $\|$ & - \\
\hline 2 & Female & 35 & 31.1 & - & L3-4 & Extrusion & 5 & Annulotomy & ॥ & - \\
\hline 3 & Female & 41 & 28.3 & - & $\lfloor 4-5$ & Sequestration & 7 & Natural & III & - \\
\hline 4 & Female & 35 & 28.0 & - & L5-S1 & Protrusion & 8 & Annulotomy & I & - \\
\hline 5 & Male & 32 & 23.7 & - & $\llcorner 4-5$ & Extrusion & 6 & Annulotomy & $\|$ & - \\
\hline 6 & Female & 47 & 26.2 & DM & L5-S1 & Extrusion & 5 & Annulotomy & IV & - \\
\hline 7 & Male & 42 & 30.1 & - & L4-5 & Extrusion & 6 & Natural & $\|$ & - \\
\hline 8 & Male & 26 & 28.9 & - & $\llcorner 4-5$ & Sequestration & 6 & Natural & $\|$ & - \\
\hline 9 & Female & 31 & 27.2 & - & L3-4 & Extrusion & 8 & Annulotomy & I & - \\
\hline 10 & Female & 29 & 25.7 & - & L4-5 & Sequestration & 10 & Natural & $\|$ & - \\
\hline 11 & Female & 35 & 23.9 & HTN & L5-S1 & Extrusion & 6 & Annulotomy & III & - \\
\hline 12 & Male & 36 & 29.1 & - & $\llcorner 4-5$ & Extrusion & 5 & Natural & III & - \\
\hline 13 & Female & 30 & 24.3 & - & L4-5 & Extrusion & 5 & Annulotomy & $\|$ & - \\
\hline 14 & Male & 31 & 24.8 & - & L5-S1 & Protrusion & 6 & Annulotomy & $\|$ & - \\
\hline 15 & Female & 35 & 30.6 & DM & $\lfloor 4-5$ & Sequestration & 9 & Natural & IV & - \\
\hline 16 & Female & 34 & 27.3 & - & L4-5 & Extrusion & 7 & Annulotomy & IV & - \\
\hline 17 & Male & 38 & 28.6 & DM & L5-S1 & Sequestration & 12 & Natural & III & - \\
\hline 18 & Female & 29 & 27.4 & - & L4-5 & Extrusion & 7 & Annulotomy & $\|$ & - \\
\hline 19 & Male & 34 & 29.7 & - & L5-S1 & Extrusion & 8 & Natural & IV & - \\
\hline
\end{tabular}

Degree of disc degeneration was classified by Pfirrmann et al. method. Size of AF tear was measured indirectly by the reference of tip length (10 $\mathrm{mm}$ ) of 90 degree curette. Type of AF tear was assorted by either natural, which means AF was naturally ruptured without annulotomy, or annulotomy, which was annulotomized by stab incision using surgical knife at the AF.

$\mathrm{BMI}$, body mass index; LDH, lumbar disc herniation; AF, annulus fibrosus; DM, diabetes mellitus; HTN, hypertension.

Table 3. Summarization of clinical outcomes

\begin{tabular}{|c|c|c|c|c|}
\hline \multirow{2}{*}{ Time } & \multicolumn{4}{|c|}{ Parameters of clinical outcome } \\
\hline & VAS & $p$-value & ODI & $p$-value \\
\hline Preoperative & $8.8 \pm 1.2$ & - & $73.4 \pm 5.6$ & - \\
\hline \multicolumn{5}{|l|}{ Postoperative } \\
\hline Immediate & $2.1 \pm 0.7$ & $<0.01$ & $23.1 \pm 3.7$ & $<0.01$ \\
\hline $3 \mathrm{mo}$ & $1.6 \pm 0.3^{\mathrm{a})}$ & $<0.01$ & $17.1 \pm 2.4^{\text {a) }}$ & $<0.01$ \\
\hline $6 \mathrm{mo}$ & $1.1 \pm 0.3^{\mathrm{a})}$ & $<0.01$ & $10.2 \pm 1.9^{\mathrm{a})}$ & $<0.01$ \\
\hline $12 \mathrm{mo}$ & $0.8 \pm 0.5^{\mathrm{a})}$ & $<0.01$ & $10.1 \pm 2.3^{\mathrm{a})}$ & $<0.01$ \\
\hline $24 \mathrm{mo}$ & $0.8 \pm 0.5^{\mathrm{a})}$ & $<0.01$ & $8.3 \pm 1.2^{\text {a) }}$ & $<0.01$ \\
\hline $36 \mathrm{mo}$ & $0.6 \pm 0.4^{\text {a) }}$ & $<0.01$ & $8.7 \pm 1.6^{\text {a) }}$ & $<0.01$ \\
\hline
\end{tabular}

VAS, visual analogue scale; ODI, Oswestry disability index. a) $p<0.05$.

tion, perennial back/leg pain, and neurologic deficits, as well as socioeconomic troubles resulting from substantial health care costs $[1,4,5]$. Hence, understanding the causative factors and finding ways to overcome the problem 
Table 4. Summarized data of radiographic outcome

\begin{tabular}{lccc} 
Time & Presence of peripheral hollow & Disc height anterior/posterior & $p$-value \\
Preoperative & - & & \\
\hline Postoperative & & $3.1 \pm 1.1$ & 0.42 \\
\hline Immediate & - & $3.0 \pm 0.8$ & 0.36 \\
\hline $3 \mathrm{mo}$ & - & $3.1 \pm 0.6$ & 0.23 \\
\hline $6 \mathrm{mo}$ & - & $2.8 \pm 1.1$ & 0.09 \\
\hline $1 \mathrm{yr}$ & - & $2.8 \pm 1.0$ & 0.08 \\
$2 \mathrm{yr}$ & - & $2.4 \pm 1.3$ & 0.08 \\
\hline $\mathrm{yr}$ & - & & \\
\hline
\end{tabular}

Values are presented as mean \pm standard deviation.

are critical to optimizing surgical outcomes, but efforts to devise a surgical technique have been rare to date.

To date, numerous reports have demonstrated the risk factors of recurrence following LD surgery, including the male gender, young age, the degeneration degree of the affected disc, traumatic events, and smoking [1-8]. Furthermore, some clinicians suggested that the degree of annular closure or healing following LD was associated with recurrence $[5,7,8]$. Although annular healing might be related to the disc recurrence after LD, improving the surgical technique for the closure or healing of the annular defect has only been discussed. Based on this knowledge, the authors focused on whether an unhealed AF might be directly related to recurrence, and how to improve recurrence from the point of view of the spinal surgeon. Previous reports have demonstrated that the location of the rLDH following LD commonly occurs at the annulotomized or ruptured AF site [4,6-8]. Considering this, either the spontaneously ruptured AF site in TLE, or the AF annulotomy site in SLE, do not naturally heal in some patients, which has not been widely recognized [6-8]. This may be related to rLDH following LD. Moreover, repetitive tensile load to the injured AF resultss in damage to disc tissue, which is expressed as loss of normalized peak stress and plastic deformation $[11,13]$. Consequently, several physicians have strongly advocated the development of novel surgical techniques to prevent rLDH, and to decrease morbidity and health care costs. New techniques for AR following LD have been introduced, but have not been thoroughly investigated. This study showed that AR using the easily available PushLock implant could provide favorable clinical and radiological outcomes with no recurrence, clinical deterioration, or aggravating radiologic parameters three years after surgery. Additionally, these results suggest that, if proper candidates are selected, this technique can be a valuable adjunct to reduce the incidence of rLDH and optimize surgical outcomes.

To date, only a few techniques for AR have been reported, including simple suture, crossed suture, and modified purse-string suture with conventional or newly designed implants $[10,11,13]$. However, these techniques proved to be difficult to apply during real surgery due for several reasons. First, the surgical field during discectomy was so confined and deeply-seated that conventional instrumentation (i.e., a short and thick apparatus) could not be utilized properly, especially when a minimal skin incision was used, as in the present study. Second, the conventional technique required making a knot located over the AF, which necessarily protruded into the spinal canal. This could continuously irritate and compress the nerve root and dura, causing pain similar to that caused by the preoperative herniated disc. Moreover, the irritation and compression caused by the knot could be exacerbated over time. Perineural tissues around the knot could intermingle with the knot through fibrosis formation and adhesion, precipitating an inflammatory cascade that could cause the mingled knot to enlarge and produce annoying symptoms requiring further operative interventions. Third, conventional approaches do not provide sufficient mechanical strength to withstand intradiscal pressure and tensile power loaded on the AF during normal and physical activity, as previously determined $[9,10]$.

Hence, this study evaluated PushLock, which has been widely used in other orthopedic surgeries, but not in spinal surgery, for AR following discectomy. There have 
been no previous studies on this application of Pushlock. Theoretically, the particular features of the implant are appropriate for AR. First, the implant is a spindle shape, which is exactly suited to the confined and deeplyseated surgical field of LD. Second, the implant can be positioned so that the knot is within the bony surface to which the AF attaches. Thus, neither knots nor implants will irritate or compress the dura or nerve root. Third, this suture system can provide sufficient mechanical stability against intradiscal pressure and tensile strength [14-19]. Considering that previous biomechanical studies have revealed the strain strength of the posterior AF to be approximately 1 to $3 \mathrm{MPa}$ during normal activity or exercise, and the implant could bear a pull-out strength of approximately 3 to $5 \mathrm{MPa}$ after rotator cuff repair, we hypothesized that knotless sutures with PushLock would provide sufficient stability to the repaired AF.

It is critical to choose an optimal entry-point for the PushLock so it is anchored well enough to withstand pull-out strength. Generally, as seen in the sagittal CT of the vertebral body of the lumbar spine, the cortical thickness of the posteroinferior or posterosuperior corner of the vertebral body is more prominent than others, such as the lower endplate and anterior cortex. Consequently, this study sought to identify and use the best position for insertion in all cases. To do this, the implant entry point should be placed close to the spontaneously ruptured or AF annulotomy site. In other words, the location of the rupture is also critical, and this technique might be inapplicable in cases where annulus rupture is located near the middle of the AF, height-wise, instead of at either end.

As with any study, this has several limitations. First, the biomechanical strength of PushLock has not been thoroughly examined during normal and physical activity. It is difficult to accurately extrapolate data from the available literature on the anchor suture tensile or pull-out strength/strain in AF repair. However, as described above [14-19], previous biomechanical studies have revealed that the strain strength of posterior AF is approximately 1 to $3 \mathrm{MPa}$. Other studied have demonstrated that sutures with PushLock can withstand a pull-out strength of approximately 3 to $5 \mathrm{MPa}$. Based on these results, we hypothesize that the anchor suture could provide sufficient stability to the repaired AF. A further study to evaluate the performance strength of the repair site will be undertaken in the future. Second, the PushLock implant may not be suitable for patients with severe osteoporosis, degenerative changes that result in a large osteophyte at the posterior vertebral body, ossified posterior longitudinal ligament, or for other reasons. Additionally, as described previously, if the injured site is located centrally in the AF height-wise, this technique cannot be utilized. For these excluded conditions, another new technique or modification is needed.

Despite these limitations, this study has important strengths. This is a rare inquiry into AR with the PushLock implant, a surgical approach that reduces recurrent herniation following LD. Additionally, a novel application of the PushLock system was used to determine its feasibility for real surgical application in LD, and its role in advancing the concept of AR. If proper candidates are selected, this surgical technique can be a valuable option to reduce the incidence of rLDH while optimizing surgical outcomes. The ideal candidates for AR using this technique are (1) young patients with SLE or (2) patients whose rupture site is near both ends of the AF in the TLE. However, this technique is not indicated for patients whose rupture site is at the center of AF, height-wise, or those who present with severe degenerative/frayed AF margins. Further study with a larger sample size and longer follow-up is needed to identify ideal candidates and criteria.

\section{Conclusions}

Additional biomechanical and clinical investigations are needed. Moreover, after evaluating the implant used in all patients who underwent lumbar discectomy, comprehensive individual considerations including age, level of rupture site, presence of osteoporosis, and others are needed to minimize complications. Nevertheless, the present study demonstrated the feasibility of knotless suture with the PushLock implant for AR following LD. Thus, we recommend that the PushLock implant be used as an adjunct to LD as a valuable alternative to optimize clinical results and minimize the recurrence rate if the procedure is performed in proper candidates.

\section{Conflict of Interest}

No potential conflict of interest relevant to this article was reported. 


\section{References}

1. Carragee EJ, Spinnickie AO, Alamin TF, Paragioudakis S. A prospective controlled study of limited versus subtotal posterior discectomy: short-term outcomes in patients with herniated lumbar intervertebral discs and large posterior anular defect. Spine (Phila Pa 1976) 2006;31:653-7.

2. Lebow RL, Adogwa O, Parker SL, Sharma A, Cheng J, McGirt MJ. Asymptomatic same-site recurrent disc herniation after lumbar discectomy: results of a prospective longitudinal study with 2-year serial imaging. Spine (Phila Pa 1976) 2011;36:2147-51.

3. Fountas KN, Kapsalaki EZ, Feltes CH, et al. Correlation of the amount of disc removed in a lumbar microdiscectomy with long-term outcome. Spine (Phila Pa 1976) 2004;29:2521-4.

4. McGirt MJ, Ambrossi GL, Datoo G, et al. Recurrent disc herniation and long-term back pain after primary lumbar discectomy: review of outcomes reported for limited versus aggressive disc removal. Neurosurgery 2009;64:338-44.

5. Ambrossi GL, McGirt MJ, Sciubba DM, et al. Recurrent lumbar disc herniation after single-level lumbar discectomy: incidence and health care cost analysis. Neurosurgery 2009;65:574-8.

6. Suk KS, Lee HM, Moon SH, Kim NH. Recurrent lumbar disc herniation: results of operative management. Spine (Phila Pa 1976) 2001;26:672-6.

7. Miwa S, Yokogawa A, Kobayashi T, et al. Risk factors of recurrent lumbar disc herniation: a single center study and review of the literature. J Spinal Disord Tech 2013 Nov 8 [Epub]. http://dx.doi.org/10.1097/ BSD.0b013e31828215b3.

8. McGirt MJ, Eustacchio S, Varga P, et al. A prospective cohort study of close interval computed tomography and magnetic resonance imaging after primary lumbar discectomy: factors associated with recurrent disc herniation and disc height loss. Spine (Phila Pa 1976) 2009;34:2044-51.

9. Chiang YF, Chiang CJ, Yang CH, et al. Retaining intradiscal pressure after annulotomy by different annular suture techniques, and their biome- chanical evaluations. Clin Biomech (Bristol, Avon) 2012;27:241-8.

10. Chiang CJ, Cheng CK, Sun JS, Liao CJ, Wang YH, Tsuang $\mathrm{YH}$. The effect of a new anular repair after discectomy in intervertebral disc degeneration: an experimental study using a porcine spine model. Spine (Phila Pa 1976) 2011;36:761-9.

11. Iatridis JC, MaClean JJ, Ryan DA. Mechanical damage to the intervertebral disc annulus fibrosus subjected to tensile loading. J Biomech 2005;38:557-65.

12. Pfirrmann CW, Metzdorf A, Zanetti M, Hodler J, Boos N. Magnetic resonance classification of lumbar intervertebral disc degeneration. Spine (Phila Pa 1976) 2001;26:1873-8.

13. Chan SC, Gantenbein-Ritter B. Intervertebral disc regeneration or repair with biomaterials and stem cell therapy: feasible or fiction? Swiss Med Wkly 2012;142:w13598.

14. Sawyer GA, Anderson BC, Paller D, Schiller J, Eberson CP, Hulstyn M. Biomechanical analysis of suture bridge fixation for tibial eminence fractures. Arthroscopy 2012;28:1533-9.

15. Pietschmann MF, Gulecyuz MF, Fieseler S, et al. Biomechanical stability of knotless suture anchors used in rotator cuff repair in healthy and osteopenic bone. Arthroscopy 2010;26:1035-44.

16. Barber FA, Hapa O, Bynum JA. Comparative testing by cyclic loading of rotator cuff suture anchors containing multiple high-strength sutures. Arthroscopy 2010;26:S134-41.

17. Chu T, McDonald E, Tufaga M, Kandemir U, Buckley J, Ma CB. Comparison of completely knotless and hybrid double-row fixation systems: a biomechanical study. Arthroscopy 2011;27:479-85.

18. Ruiz-Suarez M, Aziz-Jacobo J, Barber FA. Cyclic load testing and ultimate failure strength of suture anchors in the acetabular rim. Arthroscopy 2010;26:762-8.

19. Jarrett CD, McGillivary GR, Hutton WC. The $2.5 \mathrm{~mm}$ PushLock suture anchor system versus a traditional suture anchor for ulnar collateral ligament injuries of the thumb: a biomechanical study. J Hand Surg Eur Vol 2010;35:139-43. 